OPEN@ACCESS

\title{
ORNITOLOGÍA
}

COMUNICACIÓN CORTA/SHORT COMMUNICATION

\section{Primeros registros de Oxyura jamaicensis andina en Ecuador}

\author{
William A. Arteaga-Chávez \\ Universidad Central del Ecuador, Facultad de Ciencias Biológicas, Carrera de Biología. Quito, Ecuador. \\ E-mail: arteagawilliam95@yahoo.es \\ Editado por/Edited by: Elisa Bonaccorso \\ Recibido/Received: 19 enero 2019 Aceptado/Accepted: 10 marzo 2020 \\ Publicado en línea/Published online: 04 julio 2020
}

First records of Oxyura jamaicensis andina in Ecuador

\begin{abstract}
Ruddy Duck Oxyura jamaicensis andina occurs in the Central Andes and the Altiplano Cundiboyacense, in Colombia. It represents an intermediate plumage between $O$. j. jamaicensis and $O$. j. ferruginea, with a marked individual variation in plumage pattern throughout its range, which has resulted in uncertainty about its taxonomic validity. In this note, I report the first records of $O . j$. andina in Ecuador, extending its distribution range by $c .250-650 \mathrm{~km}$ southwards, within the distribution range of $O$. $j$. ferruginea.
\end{abstract}

Keywords: Andes, distribution, hibridization zone, Oxyura jamaicensis ferruginea, range overlap.

\section{Resumen}

El Pato Rojizo Oxyura jamaicensis andina se distribuye en los Andes Centrales y el Altiplano Cundiboyacense, en Colombia. Representa un plumaje intermedio entre $O$. j. jamaicensis y $O . j$. ferruginea, con una marcada variación individual en el patrón de plumaje en toda su área de distribución, que ha derivado en que se cuestione su validez taxonómica. En esta nota informo sobre los primeros registros de $O . j$. andina en Ecuador, extendiendo su área de distribución en $c$. 250-650 km hacia el sur, dentro del ámbito de distribución de $O$. j. ferruginea.

Palabras clave: Andes, distribución, zona de hibridación, Oxyura jamaicensis ferruginea, solapamiento de distribución.

El Pato Rojizo Oxyura jamaicensis comprende tres subespecies distribuidas desde Canadá hasta Tierra de Fuego (Chile), en humedales desde el nivel del mar hasta 4500 m de altitud (Adams \& Slavid, 1984; Fjeldså \& Krabbe, 1990; Brua, 2001). La subespecie O. j. jamaicensis se encuentra desde el sur de Canadá hasta América Central y el Caribe, $O . j$. ferruginea se encuentra desde el sur de Colombia hasta Tierra del Fuego y $O . j$. andina es endémica de los Andes Centrales y el altiplano Cundiboyacense de Colombia (Lehmann, 1946; Muñoz-Fuentes et al., 2013; Lozano-Jaramillo et al., 2018).

El estatus taxonómico de $O . j$. andina ha sido cuestionado, considerándose una forma intermedia o híbrida entre $O$. $j$. jamaicensis y $O$. $j$. ferruginea por su plumaje facial intermedio entre las mejillas blancas de $O . j$. jamaicensis y las mejillas negras de O.j. ferruginea (Todd, 1979; Fjeldså, 1986). La variación individual en todo el rango de $O . j$. andina sugiere que su área de distribución es una zona de hibridación antigua y amplia (Fjeldså, 1986; Livezey, 1995). Por este patrón de plumaje intermedio entre O.j.jamaicensis y O.j.ferruginea, se ha argumentado que las tres formas representan una sola especie (McCracken \& Sorenson, 2005; MuñozFuentes et al., 2013; Donegan et al., 2015). Otros autores han sugerido separar O. jamaicensis y O. ferruginea, pero invalidar la subespecie $O . j$. andina debido a su supuesto origen híbrido (Livezey, 1995; Carboneras \& Kirwan, 2019; del Hoyo et al., 2019). Por el contrario, Lehmann (1946) y Clements et al. (2018) consideran a $O$. j. andina como una subespecie válida de $O$. jamaicensis por sus características morfológicas. Sin embargo, un estudio genético concluyó que $O . j$. andina podría ser considerada como una especie completa, debido a que la población de $O . j$. andina es genéticamente distinta de las poblaciones de $O . j$. jamaicensis y $O . j$. ferruginea 
(Lozano-Jaramillo et al., 2018). Debido a esta controversia taxonómica es importante colectar y publicar nueva información de campo sobre la distribución en las zonas de contacto entre $O . j$. andina y $O$. j. ferruginea. En este manuscrito se adopta el tratamiento de subespecie para O.j. andina (Lehmann, 1946; Todd, 1979; Fjeldså, 1986; Clements et al., 2018).

Los machos de Oxyura jamaicensis andina se distinguen de $O$. j. jamaicensis y $O$. j. ferruginea por presentar una marcada variación de plumaje facial en toda su área de distribución. Los machos presentan patrones individuales en el plumaje de las mejillas, que varía entre diseños de líneas oblicuas que pueden formar una mancha blanca casi sólida en algunos individuos, mientras que otros muestran manchas irregulares pequeñas y dispersas (Adams \& Slavid, 1984, Fjeldså \& Krabbe, 1990).

Oxyura jamaicensis andina se distribuye entre 2500-4000 m s.n.m (Ayerbe-Quiñones, 2018). Habita principalmente en humedales y lagunas con delgadas capas de vegetación flotante (e.g., Salviniaceae y Araceae; Van der Hammen et al., 2008). Se alimenta de semillas, raicillas, insectos, crustáceos y otros invertebrados acuáticos (del Hoyo et al., 2019). Actualmente se considera En Peligro de extinción en Colombia, debido principalmente a la pérdida de hábitat, cacería y contaminación (Renjifo et al., 2016).

En esta nota presento los primeros registros de $O . j$. andina en Ecuador (cf. Freile et al., 2019), obtenidos entre septiembre de 2016 y agosto de 2018 en siete humedales andinos (Figura 1). A continuación, se presentan los registros por localidad y en orden cronológico, con un total de 10 observaciones obtenidas a partir de visitas esporádicas de campo y mediante información que ha sido compartida por otros observadores de aves. La identificación de hembras se basó en el macho acompañante, por lo que es posible que correspondan también a O.j. ferruginea (ver más adelante).

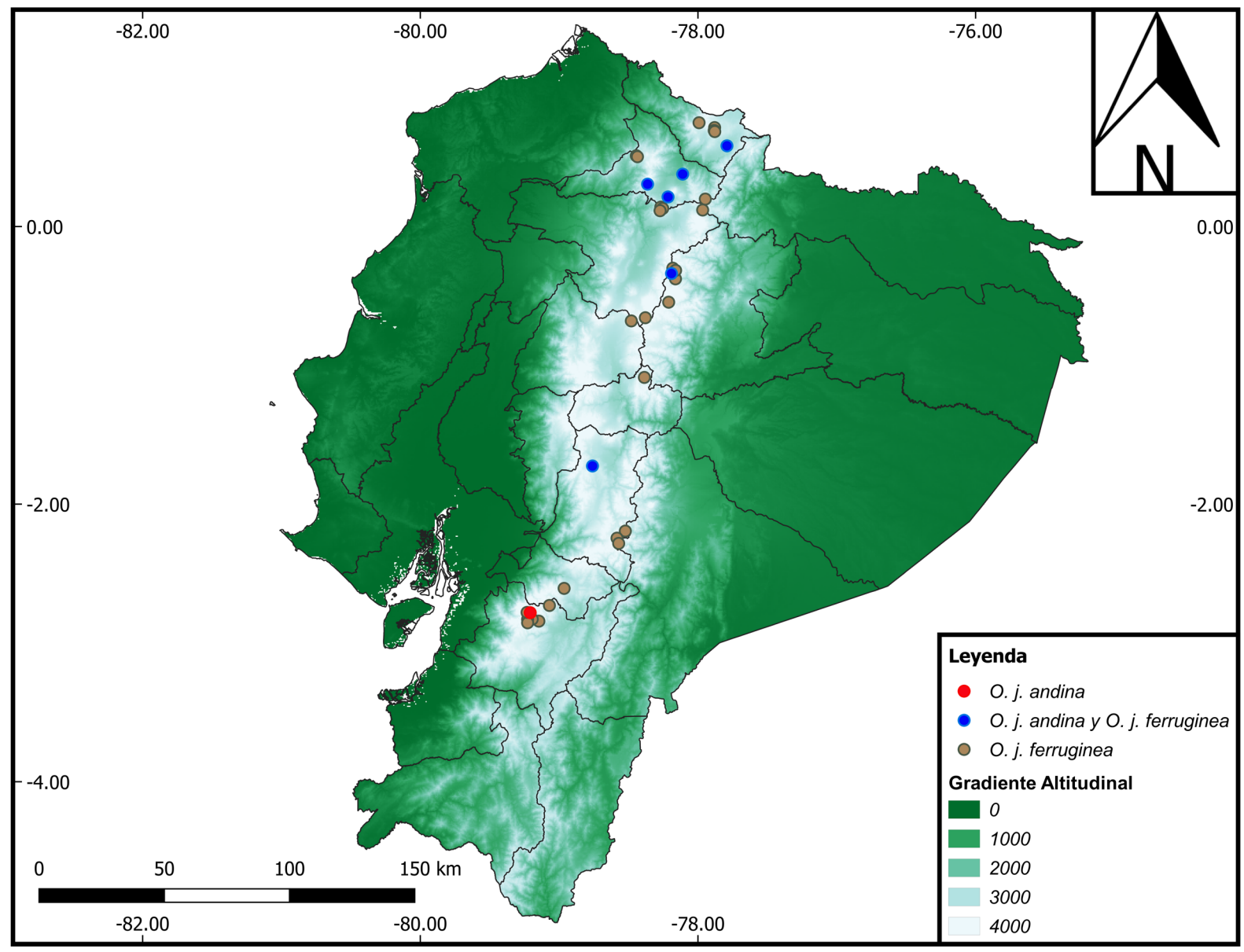

Figura 1: Localidades de observación y solapamiento de Oxyura jamaicensis andina y $O$. j. ferruginea en los Andes del Ecuador. 


\section{Yahuarcocha, provincia de Imbabura (0,37756, -78,11015; 2210 m s.n.m.)}

El 4 de septiembre de 2016 se observó una pareja de $O . j$. andina descansando en el espejo de agua en la orilla oeste de la laguna, cerca de totorales (Schoenoplectus californicus). Mantenían su cuello y cabeza recogidos hacia un costado y permanecían aislados de otros grupos de aves acuáticas. Dos días más tarde, se encontró cuatro individuos en el mismo lugar (tres machos y una hembra), que nadaban y se zambullían junto a un grupo de $O$. j. ferruginea. Dos machos tenían una pequeña mancha blanca poco definida en la región malar y otro presentaba un parche blanco conspicuo en sus mejillas. La documentación fotográfica y de vídeo de este registro fueron reportados al Comité Ecuatoriano de Registros Ornitológicos (CERO; Freile et al., 2019) (Figura 2A).

El 9 de julio de 2017, se registraron cinco individuos (una pareja con una cría y dos machos) $300 \mathrm{~m}$ al norte de las primeras observaciones, cerca de la orilla, en pequeños parches de totora. Dos machos se movían entre un grupo disperso de $O$. j. ferruginea, mientras que la pareja con una cría permanecía aislada. Los adultos vigilaban de cerca a su cría mientras se zambullía y forrajeaba bajo el agua (Figura 2B). Posteriormente, se observó a un macho de $O$.j. ferruginea acercarse hacia la pareja, pero este fue desplazado por el macho adulto de $O$. j. andina. El 7 de abril de 2018 se observaron dos machos de $O$. j. andina en el mismo lugar de la anterior observación. Se movían solos entre un grupo de $O$. j. ferruginea, mostrando un comportamiento de confrontación o rechazo ante individuos de $O . j$. ferruginea con movimiento de cabeza hacia arriba y abajo. El 21 de abril de 2018, se observaron tres individuos machos de $O . j$. andina en el mismo sector, descansando junto a dos hembras. Dos de estos individuos correspondían a registros previos. Una hembra cuya identidad subespecífica no pude determinar se acercó al grupo, pero fue rechazada por un macho en dos ocasiones (Figura 2C). El 10 de mayo de 2018, Y. Luna (com. pers.) registró en esta localidad una pareja de $O . j$. andina descansando con el cuello y cabeza recogidos a un costado cerca de un grupo de $O$. j. ferruginea. Un macho de $O$. j. ferruginea se acercó agresivamente a confrontar a los dos individuos de $O . j$. andina, los cuales respondieron con sonidos nasales mientras abrían y cerraban el pico y movían la cabeza hacia adelante y atrás. Ante el aparente dominio de $O . j$. ferruginea, los individuos de $O . j$. andina se zambulleron y buscaron refugio en los totorales (Figura 2D).

\section{EI Salado, provincia del Carchi, (0,58159, -77,79111; 2760 m s.n.m.)}

En marzo de 2017, J. M. Loaiza (com. pers.) observó un individuo macho de $O . j$. andina moviéndose cerca de individuos de $O$. j. ferruginea.

\section{San Pablo, provincia de Imbabura (0,21324, -78,21561; 2650 m s.n.m.)}

El 7 de abril de 2018 se observó un macho de $O . j$. andina cerca de un grupo de $O$. $j$. ferruginea que se encontraban descansando en un parche de jacintos de agua (Eichhornia crassipes) en la orilla este del lago. Posteriormente, el grupo fue ahuyentado por unos pescadores y empezó a dispersarse. El individuo de $O . j$. andina se aproximó directamente a una pareja de $O$. j. ferruginea, pero el macho reaccionó irguiendo su cola, encorvando su cuello y cabeza, y confrontándolo. En respuesta a estos ataques, el individuo de $O . j$. andina se zambulló y se alejó.

El 5 de mayo de 2018, J. Chandi y Y. Luna (com. pers.) registraron tres machos de $O$. j. andina entre un grupo disperso de $O . j$. ferruginea. Observaron que los individuos de $O . j$. ferruginea mostraban un comportamiento de rechazo ante $O . j$. andina, similar al descrito anteriormente (Figura 2E).

\section{Cuicocha, provincia de Imbabura (0,30548, -78,36293; 3068 m s.n.m)}

El 29 de abril de 2018 se observó un macho de $O . j$. andina moviéndose en el canal El Ensueño, que separa los islotes Wolf y Yerovi. Estuvo alimentándose cerca a individuos de Focha Andina Fulica ardesiaca, aislado de individuos de O.j. ferruginea, que también se encontraban en este humedal (Figura 2F).

\section{Sucus, provincia de Napo (-0,33841, -78,19063; 3930 m s.n.m)}

El 25 y 26 de mayo de 2018 se observó un individuo macho de $O$. j. andina junto a un grupo disperso de $O . j$. ferruginea. En ambos días, se observaron dos individuos de $O . j$. andina que en ocasiones permanecían con algunas hembras, mismas que también se juntaban con machos de $O$. j. ferruginea. Este comportamiento, no permitió determinar si había una pareja de $O$. j. andina en esta laguna (Figura $2 \mathrm{G}$ ).

\section{Colta, provincia de Chimborazo (-1,72504, -78,75975; 3180 m s.n.m.)}

El 28 de febrero de 2018 se observaron cinco individuos de $O . j$. andina: dos machos cerca de un grupo de $O$. j. ferruginea en la orilla norte de la laguna, y una pareja con una cría, que permanecía aislada en la orilla noreste (Figura 2H). 


\section{Laguna Patoquinoas, provincia de Azuay (-2.782045, -79.208416; 3810 m s.n.m.)}

El 7 de agosto de 2018, J. Chandi (com. pers.) observó y fotografió a dos individuos machos de $O$. j. andina que se encontraban descansando en el espejo de agua (Figura 2I). Durante el tiempo de observación no se registró a $O$. j. ferruginea.

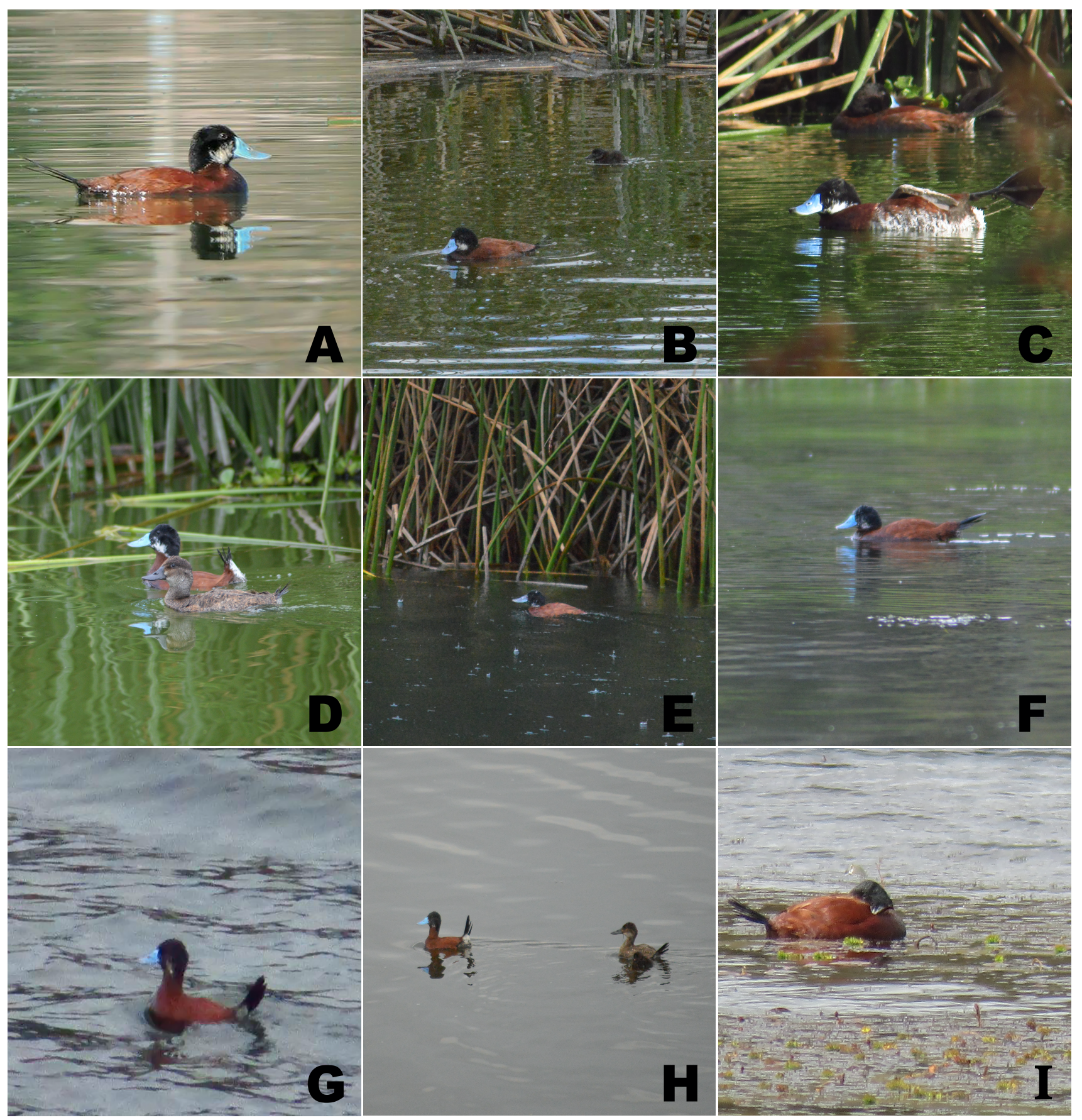

Figura 2: Registros fotográficos de Oxyura jamaicensis andina en humedales andinos de Ecuador. Los fotografos se mencionan junto a la fecha de cada registro. Yahuarcocha, provincia de Imbabura: (A) 6 de septiembre de 2016, Eduardo Obando; (B) 9 de julio de 2017, William Arteaga-Chávez; (C) 21 de abril de 2018, Dayana Togán; (D) 10 de mayo de 2018, Yolanda Luna. Cuicocha, provincia de Imbabura: (E) 29 de abril de 2018, William Arteaga-Chávez. San Pablo, provincia de Imbabura: (F) 5 de mayo de 2018, Yolanda Luna. Sucus, provincia de Napo: (G) 25 de mayo de 2018, Dayana Togán. Colta, provincia de Chimborazo: (H) 28 de febrero de 2018, William Arteaga-Chávez. Patoquinoas, provincia de Azuay: (I) 6 de agosto de 2018, Javier Chandi. 
Los machos observados de $O . j$. andina en las siete localidades de Ecuador mostraron una marcada variación individual en el diseño de mejillas blancas. En el norte (Yahuarcocha, San Pablo y Cuicocha) presentaron parches blancos más conspicuos, de formas irregulares, mientras que los individuos más australes (Sucus, Colta y Patoquinoas), presentaron manchas pequeñas dispersas. Esta variación es similar a lo que ocurre en los Andes Centrales y Orientales de Colombia (Adams \& Slavid, 1984; Fjeldså, 1986), aunque en Colombia también existen machos de $O . j$. andina que tienen parches blancos grandes y uniformes, similares a los machos de $O . j$. jamaicensis.

Se ha propuesto que $O . j$. andina podría considerarse como una especie y que la variabilidad fenotípica existente en la población no es el resultado de la afluencia reciente de individuos de otras poblaciones (Lozano-Jaramillo et al., 2018). Sin embargo, los individuos registrados en Ecuador reflejan evidente variación, con fenotipos más cercanos a $O . j$. ferruginea, que tiene cabeza y cuello completamente negros y es la subespecie previamente registrada en Ecuador (Ridgely \& Greenfield, 2006; McMullan \& Navarrete, 2017). La variabilidad en el patrón de manchas blancas podría reflejar que hacia los extremos de su área de distribución muestran características más parecidas a las formas parentales. Así, las poblaciones de Colombia, hacia el norte, mantienen características más puras de $O . j$. andina, mientras que en las poblaciones más al sur, estas características empiezan a perderse gradualmente, adquiriendo características fenotípicas de $O$. j. ferruginea (Fjeldså, 1986; McCracken \& Sorenson, 2005).

Estos registros de $O . j$. andina son los primeros en Ecuador, extienden su área de distribución en $c$. 250-650 km hacia el sur, tomando en cuenta el registro más norteño en El Salado y el más austral en Patoquinoas. Esta nueva área de distribución, y probable zona de hibridación, se sobrepone en gran parte con el área de distribución de O. j. ferruginea en Ecuador (Freile \& Restall, 2018). Además, los reportes de Yahuarcocha, a 2200 m s.n.m, representan el registro a menor altitud (ver Ayerbe-Quiñones, 2018). La regularidad de los muestreos realizados en Yahuarcocha entre 2016 y 2018 sugiere que existe una población residente de $O$. j. andina que coexiste con O. j. ferruginea.

Dada la coexistencia de las dos subespecies en Ecuador, es probable que individuos de $O . j$. andina estén reproduciéndose con individuos de $O$. j. ferruginea. Se observaron dos parejas con una cría, integradas por un macho de $O . j$. andina y una hembra que podría corresponder a cualquiera de las dos subespecies, una en Yahuarcocha (Figura 2B) y otra en Colta (Figura 2H). La diferenciación entre subespecies en los machos es evidente, pero las hembras son muy parecidas (Fjeldså, 1986), por lo que estas crías podrían corresponder a una generación híbrida entre las dos subespecies. Esta similitud no permitió determinar si existía una preferencia selectiva de pareja por parte de los machos de $O . j$. andina.

Existen registros de $O . j$. andina en el sur de Colombia, en el área de distribución regular de $O . j$. ferruginea en ese país. En la laguna La Cocha, departamento de Nariño (1,10204, -77,14946; 2760 m.s.n.m), A. Mendoza y R. Fernández (com. pers.) fotografiaron un macho. Es probable que estos individuos provengan de poblaciones que ocurren regularmente en el sur de los Andes Centrales de Colombia, cuyo desplazamiento hacia el sur se deba a un posible crecimiento lento de la población. La Cocha se encuentra en la vertiente oriental del nudo de Los Pastos o Macizo Colombiano, donde divergen los Andes colombianos hacia el norte, y hacia el sur continúan los Andes ecuatorianos (Kattan et al., 2004), que podría ser la ruta más favorable de dispersión.

Los datos recopilados en esta nota abren la oportunidad de estudiar las interacciones de estas dos subespecies ahora sintópicas, para determinar si existe dominancia de individuos de mejillas negras hacia individuos de mejillas blancas. En general, se observaron individuos de $O . j$. andina con grupos pequeños de $O . j$. ferruginea, aunque sin formar congregaciones, más bien forrajeando y descansando aisladamente. También se observaron interacciones agresivas de machos de $O . j$. ferruginea hacia machos de $O . j$. andina. Estos comportamientos podrían darse en respuesta a disputas territoriales (Siegfried, 1976). También es importante investigar si $O . j$. andina está ampliando su área de distribución, estableciendo poblaciones residentes en Ecuador y, por tanto, hibridando con $O . j$. ferruginea, o si se trata de una expansión temporal debido a un eventual crecimiento poblacional o a una posible migración a Ecuador en respuesta a la pérdida de hábitat en Colombia. 


\section{AGRADECIMIENTOS}

A Eduardo Obando y Yolanda Luna por sus fotografías. A la Fundación Altrópico por facilitarme equipos. A Alejandro Mendoza, Ronald Fernández, José María Loaiza y Javier Chandi por compartir sus observaciones desinteresadamente. A Dayana Togán por su ayuda en campo. A Iván Jácome y Elisa Bonaccorso por sus valiosas revisiones y comentarios. A dos revisores anónimos que realizaron importantes comentarios en versiones anteriores. A Juan Freile por su paciencia y sugerencias durante la elaboración de este trabajo, que ayudaron sustancialmente a la estructuración del mismo.

\section{REFERENCIAS}

Adams, J., \& Slavid, E. R. (1984). Cheek plumage pattern in Colombian ruddy duck Oxyura jamaicensis. Ibis, 126(3), 405-407. DOI: https://doi.org/10.1111/j.1474-919X.1984.tb00262.x

Ayerbe-Quiñones, F. (2018). Guía ilustrada de la avifauna colombiana. Bogotá, Colombia: Wildlife Conservation Society.

Brua, R. B. (2001). Ruddy duck (Oxyura jamaicensis). En: A. Poole, \& A. Gill (Eds.), Birds of North America. Philadelphia, USA.

Carboneras, C., \& Kirwan, G. M. (2019, Enero 19). Ruddy Duck (Oxyura jamaicensis). En J. del Hoyo, A. Elliott, J. Sargatal, D. A. Christie \& E. de Juana (Eds.), Handbook of the birds of the world alive. Barcelona, España: Lynx Edicions. URL: https://www.hbw.com/node/52934

Clements, J. F., Schulenberg, T. S., Iliff, M. J., Roberson, D., Fredericks, T. A., Sullivan, B. L., \& Wood, C. L. (2017). The eBird/Clements checklist of birds of the world: v2017. Ithaca, New York: Cornell Lab of Ornithology. URL: http://www.birds.cornell.edu/clementschecklist/download/

del Hoyo, J., Collar, N., \& Kirwan, G.M. (2019, Enero 19). Andean Duck (Oxyura ferruginea). En J. del Hoyo, A. Elliott, J. Sargatal, D. A. Christie \& E. de Juana (Eds.), Handbook of the birds of the world alive. Barcelona, España: Lynx Edicions. URL: https://www.hbw.com/node/467111

Donegan, T. M., Quevedo, A., Verhelst, J. C., Cortés-Herrera, O., Ellery, T., \& Salaman, P. (2015). Revision of the status of bird species occurring or reported in Colombia 2015, with discussion of BirdLife International's new taxonomy. Conservación Colombiana, 23, 3-48. URL: http://www.proaves.org/wp-content/uploads/2015/12/Listado-ySplits-Conservacion-Colombiana-23-3-48.pdf

Fjeldså, J. (1986). Color variation in the Ruddy Duck (Oxyura jamaicensis andina). Wilson Bulletin, 98(4), 592-594. URL: https://www.jstor.org/stable/4162312

Fjeldså, J., \& Krabbe, N. (1990). Birds of the high Andes. Copenhagen \& Svendborg, Dinamarca: Zoological Museum, University of Copenhagen \& Apollo Books.

Freile, J. F., \& Restall, R. (2018). Birds of Ecuador. London, UK: Helm Field Guides.

Freile, J. F., Solano-Ugalde, A., Brinkhuizen, D. M., Greenfield, P. J., Lysinger, M., Nilsson, J., Navarrete, L., \& Ridgely, R. S. (2019). Fourth report of the Committee for Ecuadorian Records in Ornithology (CERO) and a revision of undocumented and erroneous records in the literature. Revista Ecuatoriana de Ornitología, 5, 5279. DOI: https://doi.org/10.18272/reo.vi5.1277

Kattan, G. H., Franco, P., Rojas, V., \& Morales, G. (2004). Biological diversification in a complex region: a spatial analysis of faunistic diversity and biogeography of the Andes of Colombia. Journal of Biogeography, 31(11), 1829-1839. DOI: https://doi.org/10.1111/j.1365-2699.2004.01109.x

Lehmann, F. C. (1946). Two new birds from the Andes of Colombia. Auk, 63(2), 218-223. URL: https://www.jstor.org/stable/4080013 
Livezey, B. C. (1995). Phylogeny and comparative ecology of stiff-tailed ducks (Anatidae: Oxyurini). Wilson Bulletin, 107(2), 214-234. URL: https://www.jstor.org/stable/4163539

Lozano-Jaramillo, M., McCracken, K. G., \& Cadena, C. D. (2018). Neutral and functionally important genes shed light on phylogeography and the history of high-altitude colonization in a widespread New World duck. Ecology and Evolution, 8(13), 6515-6528. DOI: https://doi.org/10.1002/ece3.4108

McMullan, M., \& Navarrete, L. (2017). Fieldbook of the birds of Ecuador including the Galapagos Islands and common mammals. Quito, Ecuador: Ratty Ediciones.

Muñoz-Fuentes, V., Cortázar-Chinarro, M., Lozano-Jaramillo, M., \& McCracken, K. G. (2013). Stepwise colonization of the Andes by Ruddy Ducks and the evolution of novel $\beta$-globin variants. Molecular Ecology, 22(5), 1231-1249. DOI: https://doi.org/10.1111/mec.12151

McCracken, K. G., \& Sorenson, M. D. (2005). Is homoplasy or lineage sorting the source of incongruent mtDNA and nuclear gene trees in the stiff-tailed ducks (Nomonyx-Oxyura)? Systematic Biology, 54(1), 35-55. DOI: https://doi.org/10.1080/10635150590910249

Renjifo, L. M., Amaya-Villarreal, A. M., Burbano-Girón, J., \& Velásquez-Tibatá, J. (2016). Libro rojo de aves de Colombia. Volumen II: ecosistemas abiertos, secos, insulares, acuáticos continentales, marinos, tierras altas del Darién y Sierra Nevada de Santa Marta y bosques húmedos del centro, norte y oriente del país. Bogotá, Colombia: Pontificia Universidad Javeriana \& Instituto Alexander von Humboldt.

Ridgely, R. S., \& Greenfield, P. J. (2006). Aves del Ecuador. Quito, Ecuador: Academia de Ciencias de Philadelphia \& Fundación Jocotoco.

Siegfried, W. R. (1976). Social organization in Ruddy and Maccoa ducks. Auk, 93(3), 560-570. URL: https://www.jstor.org/stable/4084957

Todd, F. S. (1979). Waterfowl: Ducks, geese, and swans of the world. San Diego, California: Sea World Press.

Van der Hammen, T., Stiles, F. G., Rosselli, L., Chisaca, M. L., Camargo, G., Guillot, G., Useche, Y., \& Rivera, D. (2008). Protocolo de recuperación y rehabilitación ecológica de humedales en centros urbanos. Bogotá, Colombia: Secretaría Distrital de Ambiente. 